Aus der Psychiatrischen Klinik der Universität in Tübingen.

\title{
Ueber das psychische Moment bei der Neurasthenie.
}

Von Prof. R. Wollenberg.

Die Neurasthenie nimmt nicht nur in dem besonderen Grenzgebiet, auf dem Internist und Psychiater häufig zusammentreffen, den ihr zukommenden breiten Raum ein, sie kann auch ganz allgemein als eine der gegenwärtig am liebsten und häufigsten diagnostizierten Krankheiten bezeichnet werden, wobei zunächst dahingestellt bleiben mag, ob dies mit Recht oder Unrecht geschieht. Jedenfalls ist es um so dringender notwendig, den so bezeichneten Krankheitsbegriff möglichst scharf und eng zu umgrenzen; nicht als ob damit eine prinzipielle Sonderung der zur inneren Medizin und der zur Psychiatrie gehörenden Fälle erstrebt werden sollte - die Natur des Grenzgebietes schließt dies von vornherein aus und wird für gewisse Fälle die Entscheidung immer von der persönlichen Stellung und dem subjektiven Ermessen des jeweiligen Untersuchers abhängig machen -, sondern damit der genannte Begriff infolge einer zu starken Belastung mit nicht dazugehörigen Krankheitsbildern und Einzelsymptomen sich nicht ins Unbestimmte verflüchtige. Daß eine solche Gefahr besteht, daß der Neurasthenie vielfach nicht dazugehörige Krankheitserscheinungen zugerechnet werden, ist von verschiedenen Seiten schon wiederholt ausgesprochen und beklagt worden. Ich nenne von Psychiatern Kraepelin, der in der Einleitung eines die Diagnose der Neurasthenie behandelnden Vortrages ${ }^{1)}$ ausspricht, daf diese zur Sammelbezeichnung der verschiedenartigsten Zustände geworden sei und heute ihre Eindeutigkeit nicht nur im ätiologischen und klinischen Sinne, sondern namentlich auch hinsichtlich der Prognose und Behandlung vollständig verloren habe; ferner Binswanger ${ }^{2}$ ), der von einer zu weitgehenden Verallgemeinerung der Diagnose Neurasthenie spricht. Als Vertreter der inneren Kliniker sei Martius angeführt, der in seiner Pathogenese innerer Krankheiten ${ }^{3}$ ) die sich verallgemeinernde Gepflogenheit, die Neurastheniediagnose ganz kritiklos zu stellen und in diesen Sammelbegriff die heterogensten Vorgänge hineinzupferchen, darauf zurückführt, dah eine gewisse Neigung bestehe, jede funktionelle Störung an sich für neurasthenisch zu erklären.

Es ist nicht der Zweck der nachfolgenden Ausführungen, auf das Krankheitsbild der Neurasthenie mit seinen unendlich mannigfaltigen psychisch-nervösen Erscheinungen im einzelnen einzugehen. Dazu gehört eine monographische Bearbeitung, wie wir sie ja von Bouveret, Löwenfeld, Müller, v. Krafft-Ebing, von Binswanger u. a. besitzen. Ich beabsichtige vielmehr nur, ausgehend von den gegenwärtigen Anschauungen über die Pathogenese der Neurasthenie, das dabei wesentlich mitwirkende psychische Moment etwas eingehender zu beleuchten, im Anschlup daran die nosologische Stellung der Neurasthenie vom psychiatrischen Standpunkt aus nach einigen Richtungen zu erörtern und unter Verwertung eigener Beobachtungen eine Gruppe von Fällen zu besprechen, die verhältnismäßig oft zu der irrtümlichen Diagnose einer Neurasthenie Anlaf geben.

Es liegt im Wesen der so bezeichneten Krankheit begründet, daB alle Versuche einer knappen und erschöpfenden Definition so wenig befriedigend ausfallen. Die kurzen Bezeichnungen „Nervenschwäche“, „nervöse Erschöpfung“, „reizbare Schwäche "des Nervensystems" sind zwar bequem und in der Praxis beliebt, treffen aber nur eine Seite der Sache, weil sie das wichtige psychische Moment nicht deutlich genug zum Ausdruck bringen. Dieser Anforderung entspricht v. KrafftEbings ) Definition, die aber sonst sehr allgemein gehalten

1) Kraepelin. Die Diagnose der Neurasthenie. Separatabdruck. Münchener medizinische Wochenschrift 1902, No, 40, -2 ) Binswanger. Die Pathologfe und Therapie der Neurasthenie, Jena 1896, S. 340 . - 3) Martius, Pathogenese innerer Krankheiten. Ill. Funktionelle Neurosen, Leipzig.Wien 1903, S. 299.

4) v. Krafft-Ebing, Nervosität und neurasthenischeZustande. Nothnagels Spezielle Pathologie, Bd. XII, H. 2, S. 36. „Die Neurasthenie ist eine allgemeine, d. h. über sehr große Gebiete des Nervensystems verbreitete Neurose von vorwiegend zentraler (ce* rebraler) Lokalisation. mit prädominierenden psychischen Symptomen (Neuropsychose). Daneben finden sich massenhaft motori- 
ist. Es handelt sich eben. wie Bins wanger ${ }^{1}$ ) mit Recht hervorhebt, um Krankheitserscheinungen und Krankheitszustände, die einer schärferen begrifflichen Zusammenfassung infolge ihrer flüchtigen. schwankenden und wechselnden Merkmale schwer zugänglich sind. Wenn es auch eine gewisse Gruppe von Fällen gebe, die durch gemeinschaftliche, schärfer ausgeprägte und bis zu gewissem Grade stabil und gesetzmäBig auftretende Krankheitssymptome ausgezeichnet seien und als eigene Krankheitsform unter einer speziellen Krankheitsbezeichnung zusammengefaßt und diagnostiziert werden können, so lasse sich die Neurasthenie doch nicht scharf von den anderweitigen, ihr nahestehenden funktionellen Nervenkrankheiten unterscheiden. Es bestehen hier weder nach oben noch nach unten scharfe Grenzen, sodal die Neurasthenie auf der Grenz. scheide zwischen den unfertigen Neuropathien und den vollentwickelten Neurosen und Psychosen stehe. Dementsprechend gestaltet Binswanger. bei ausdrücklicher Anerkennung der Moglichkeit, sie als solche in ihrer typischen Gestaltung wohl $\mathrm{zu}$ erkennen und zu diagnostizieren, den diagnostischen Begriff der Neurasthenie fast negativ ${ }^{2}$.

Bestimmteres läßt sich über die Pathogenese der neurasthenischen Phänomene sagen. Wir gehen dabei ans von einer eigenartigen. ererbten oder früher oder später erworbenen funktionellen Yinderwertigkeit des zentralen Nervensystems, welche dieses im ganzen oder in dem einen oder anderen seiner Teile betrifft, wobei aber die corticalen Empfindungszentren unter allen Umständen als beteiligt zu denken sind. Infolgedessen kommt es zu pathologisc: :a Reaktionen zunächst im Sinne einer krankhaften Uebererregbarkeit. von denen die durch die Beteiligung des Gehirns bedingten naturgemäß von überragender Bedeutung sind und als "illegitime", d. h. durch äufiere Ursachen nicht genügend motivierte und dabei äußerst unangenehme Empfindungen (Martius) zum Bewußtsein kommen. Diese psychische Hyperästhesie oder richtiger Hyperalgesie ist schon von dem älteren Romberg, der allerdings der Terminologie seiner Zeit gemäß nicht von Neurasthenie. sondern von Hypochondrie spricht, richtig erkannt worden und wird von allen neueren Bearbeitern der Neurasthenie betont. Mit besonderer Schärfe und zwingender Logik hat neuerdings Martius auf die geradezu fundamentale Bedeutung hingewiesen, die ihr in der Pathogenese der Neurasthenie zukommt. Er sieht in dieser quantitativ und qualitativ krankhaften Abänderung der Empfindungen das eigentliche Wesen des neurasthenischen Zustandes und bezeichnet das Auftreten unangenehmer Gefühlsbetonung schon bei physiologischen, normalerweise unterbewubt und jedenfalls unterschmerzlich bleibenden Akten, wie z. B. Stoffwechselvorgängen, Blutzirkulation, Einstellung auf Temperaturunterschiede, Muskelkontraktionen etc., als neurasthenische Reaktion. Im einzelnen können diese "illegitimen" Empfindungen, die sich dem Bewußtsein ohne Zutun der Aufmerksamkeit bei irgendwelchen gleichgültigen Anlässen aufdrängen, äußerst mannigfaltig sein, indem sie als Ermüdungssensationen, als Kopfdruck, Blasen-, Eingeweide- oder sonstiger Organschmerz, als unbestimmte Angst-, Beklemmungs-, Schwindel-, Schwäche-, Ohnmachtsempfindungen auftreten; „das Wesentliche und Bleibende ist die spezifisch-neurasthenische Gefühlsbetonung" selbst, die „zu jeder Funktion, sei dieselbe an sich normal oder ihrerseits abwegig, hinzutreten" kann (Martius). ${ }^{3}$ )

Hiermit ist ein brauchbares Kriterium für die Ausscheidung gewisser neuropathischer Erscheinungen gegeben, die vielfach fälschlich zur Neurasthenie gezählt werden. Es handelt sich auch hier um angeborene, seltener erworbene funk-

sche, sensible, vasomotorische, sekretorische und selbst vegetative Funktionsstórungen, aber wesentlich ausgelöst und beeinflufst durch psychische Vorgänge und vielfach exzentrische Erscheinungen darstellend.

1) Binswanger 1. c., S. $4 \mathrm{ff}$

2) Unter Neurasthenie fassen wir die neuropathischen Krankheitserscheinungen zusammen, welche auf dem Boden einer allgemeinen funktionellen Erkrankung des Nerveusystems erwachsen sind, jedoch nicht den vollentwickelten funktionellen, dem gleichen Boden entstammenden Psychosen und Neurosen wegen ihres un. fertigen Charakters zugerechnet werden können." (Binswanger 1. c., s. 5 ).

3) i. c., s. 308 und 304. tionelle Minderwertigkeiten, die aber auf - im Verhältnis zum Gehirn - niedere nervöse Zentren beschränkt sind und infolge der in diesen bestehenden krankhaften Uebererregbarkeit zu mannigfachen Störungen, z. B. der Herztätigkeit, der motorischen, der vasomotorischen, sekretorischen, trophischen. vor allem aber der vegetativen Funktionen, führen können. Diese lokalisierten konstitutionellen Organschwächen werden naturgemäß häufig der Ausgangspunkt der Neurasthenie, sie sind aber selbst nicht neurasthenisch, wenn nicht die charakteristische, unangenehme Gefühlsbetonung hinzutritt, was nach dem Gesagten jederzeit der Fall sein kann.

Es ist begreiflich. daf diese primäre krankhafte Empfindungsstörung nicht ohne mehr oder minder tiefgreifenden Einflub auf den allgemeinen psychischen Zustand des betroffenen Individuums sein kann. Ohne jetzt hierauf einzugehen, möchte ich mich nur gegen die Annahme verwahren, als wollte ich mit der Anerkennung und starken Betonung dieses psychischen Moments die Neurasthenie zu einer Psychose im eigentlichen Sinne stempeln. Neuerdings hat $\mathrm{Erb}^{\mathrm{l}}$ ) in der Diskussion zu meinem Referat „Die nosologische Stellung der Hypochondrie ${ }^{\text {(2) }}$ Gelegenheit genommen, ausdrücklichen Protest einzulegen, „gegen die an manchen Stellen immer deutlicher hervortretende Anschauung, welche die Neurasthenie als eine Art der psychischen Erkrankung als eine ,Psychoneurose" betrachten möchte." Eine so radikale Anschaunng ist nun in psychiatrischen Kreisen doch wohl nur vereinzelt zu finden. Dies hat in jener Sitzung sofort Fürstner $r^{3}$ ) ausgesprochen, indem er sagte, er glaube nicht, daß es viele Leute gebe, welche die Neurasthenie grundsätzlich als Psychose ansehen. Aus der Literatur kann sodann Binswanger angeführt werden, der an mehreren Stellen seines oben erwähnten Buches betont, daß er durchaus nicht der Auffassung huldige, nach welcher die neurasthenischen Krankheitserscheinungen ausschließlich auf psychischen Krankheitsvorgängen beruhen oder "sämtliche im Verlauf der Neurasthenie auftretenden Beschwerden als Folgen einer cerebralen Funktionsstörung“ aufgefaßt werden müssen. Der genannte Autor ist vielmehr der Meinung, daß die auf psychischem Gebiet liegenden Symptome der Neurasthenie eine Gruppe von Krankheitserscheinungen für sich bilden und außer direktem Zusammenhang mit den körperlichen Krankheitserscheinungen stehen, daf die letzteren aber eine weitergehende Bedeutung auf den nervösen Gesamtzustand durch die der Neurasthenie zugrunde liegenden allgemeinen Störungen der nervösen Reaktionen gewinnen; und hierbei weist auch er den psychischen Vorgängen die Hauptrolle zu. - Auch Krafft-Ebing, der allerdings weiter geht. da er eine fast ausnahmslose, direkte oder indirekte, Abhängigkeit der Symptome von psychischen Vorgängen lehrt und von einer Neuropsychose spricht, hebt hervor, daß es sich dabei auf psychischem Gebiet nur um eine Elementarstörung handle. Auch er entfernt sich also mit seiner Auffassung nicht weit von der oben erwähnten, durch Martius vertretenen, da auch nach dieser eine Empfindungsanomalie, also eine psychische Elementarstörung, das Wesentliche des neurasthenischen Zustandes ausmacht. Mit dieser Anschauung, die ich für richtig halte, ist nun zwar die. prinzipielle Bedeutung des psychischen Moments für die Pathogenese der Neurasthenic anerkannt, es ist aber doch noch ein sehr weiter Schritt von hier zu den Psychosen im eigentlichen Sinne. Das Verhältnis der Neurasthenie zu diesen bedarf demnach einer besonderen Erörterung.

Die Unklarheit, die, wie bereits oben erwähnt, infolge der zu weiten Ausdehnung des Neurastheniebegriffes in diagnostischer und prognostischer Beziehung besteht, tritt besonders deutlich hervor, wenn man eine zusammenfassende Darstellung der noch in den Rahmen der Neurasthenie als solcher fallenden, ausgesprochenen psychischen Krankheitserscheinungen versucht. Es ist ja bekannt und ohne weiteres verständlich, daf das Nervensystem den Schädigungen des Individuallebens um so früher und vollständiger erliegt, mit je geringerer Widerstandskraft es ab origine ausgestattet war. Dement-

1) Erb, Diskussionsbemerkung Archıv für Psychiatrie 1905, Bd. 40, S. $1035 \mathrm{f}$. -2) Zentralblatt für Nervenheilkunde und Psychiatrie 1905, No. 193. - 3) Fürsiner 1. C. S. 1036 . 
sprechend sehen wir besonders häufig solche Menschen neurasthenisch werden, die als Zeichen einer angeborenen Konstitutionsschwäche auch ein in toto abnorm widerstandsschwaches Zentralnervensystem oder die mehr lokalisierten Zeichen nervöser Minderwertigkeit mit auf die Welt gebracht haben. Diese neuropathische Belastung pflegt sich nun auf psychi. schem Gebiet in mehr oder weniger zahlreichen Abweichungen bemerkbar zu machen, die in das weite Gebiet der konstitutionellen Nervosität und der Entartungszustände gehören und, obwohl sie gewisse Züge (z. B. abnorme Ermüdbarkeit bei Degenerierten) mit der Neurasthenie gemein haben können, von dieser doch grundsätzlich zu trennen sind. Denn wenn auch ihre Zusammenfassung in einer Lehrdarstellung, wie wir sie Binswanger verdanken, sich gewiß rechtfertigen läßt, so ergibt sich daraus doch für die Praxis ein doppeltes Bedenken, nämlich einmal, weil man leicht dazu kommen kann, die im Vergleich $\mathrm{zu}$ diesen grob auffälligen degenerativen Krankheitszeichen weniger hervortretenden psychischen Eigentümlichkeiten der "einfachen Neurasthenie“ zu unterschätzen oder gar ihr Bestehen gänzlich in Abrede zu stellen, sodann, weil, auch abgesehen von den vielfach nur aus Bequemlichkeit oder aus Unkenntnis so bezeichneten Fällen, ganz heterogene psychopathische Zustände vielfach als Neurasthenien diagnostiziert werden.

Letzteres kann nicht besser illustriert werden als durch eine Betrachtung des Verhältnisses zwischen Neurasthenie und Hypochondrie, wie es sich historisch entwickelt liat. Früher, d. h. ehe das Krankheitsbild der Neurasthenie durch Beards Arbeiten Gemeingut der Aerzte geworden war, wurden die hierher gehörigen Erscheinungen und noch einige mehr der sehr häufig zu Unrecht diagnostizierten Hypochondrie zugerechnet. Nach dem genannten Zeitpunkt wurde dann der Begriff der letzteren immer mehr eingeengt, und schließlich verschwand er fast aus der Nosologie, da die überwiegende Mehrzahl der Fachgenossen sich zu dem Satze bekannte: Eine selbständige Krankheitsform Hypochondrie gibt es nicht; was so genannt wurde, ist nur eine Teilerscheinung der Neurasthenie. In meinem auf Wunsch der Südwestdeutschen Wanderversammlung erstatteten Referat habe ich dann ausgeführt, daf der erste Teil dieses Satzes richtig sei, da die nosologische Selbständigkeit der Hypochondrie sich nicht aufrecht erhalten lasse, daß es aber nur dann gerechtfertigt sei, die Hypochondrie vollständig in der Neurasthenie aufgehen zu lassen, wenn man dem jetzt vielfach herrschenden Brauch gemäß unter der letzteren Bezeichnung nicht nur die nervöse Erschöpfung, sondern auch die Nervosität, die konstitutionellen Verstimmungen, die Zwangszustände, die psychopathischen Minderwertigkeiten etc., kurz das ganze Gebiet der Entartungszustände im weiteren Sinne verstehe. Wenn nun auch, wie oben schon bemerkt wurde, die hierher gehörigen konstitutionellen Schwächlinge das geeignetste Material für eine Erkrankung an Neurasthenie abgeben, so sind sie doch an und für sich nicht neurasthenisch, wenn man den Krankheitsbegriff auch hier so eng faßt, wie es im Interesse der Klarheit erforderlich ist, nämlich als eine von bisher Gesunden erwerbbale chronische nervöse Erschöpfung (Kraepelin). Nur für eine Hauptgruppe der sogenannten Hypochondrien läßt sich dann die Zugehörigkeit zur Neurasthenie aufrecht erhalten, nämlich für die später noch kurz zu besprechenden Fälle, bei denen in den Vordergrund tretende nosophobische Vorstellungen jener ein besonderes, hypochondrisches, Gepräge verleihen (hypochondrische Neurasthenie). Dagegen mub die zweite grobe Gruppe - von den zahlreichen hypochondrischen Episoden im Verlaufe anderer Geisteskrankheiten kann hier abgesehen werden - aus der Neurasthenie ausgeschieden und dem oben erwähnten Gebiet der Entartungszustände im weiteren Sinne zugewiesen werden. Denken wir uns z. B. folgenden Fall:

Eine weibliche Kranke, in der ersten Bälfte der 40er Jahre stehend, psychopathisch belastet, selbst menschenscheu, dabei leidenschaftlich und äußerst hartnäckig an einmal gefaßten Meinungen festhaltend, insbesondere aber von jeher zu hypochondrischer Selbstbeobachtung und laienhaft-medizinischen Ueberlegungen geneigt, außerdem an "nervösem", bei jeder Gemütserregung eintretendem, übrigens "ohne jede Störung des subjektiven Befin- dens verlaufendem Durchfall und an Enteroptose mäßigen Grades leidend, zieht sich bei bis dahin vollständigem Wohlbefinden infolge einer unzweckmäßigen Muskelanstrengung eine schmerzhafte Affektion (Muskelzerrung?) des Rückens und Leibes zu, zu welcher sich bald unangenehme Sensationen von seiten der Intestina und die immer bestimmter auftretende Ueberzeugung, an einer schweren Darmaffektion zu leiden, gesellen. Den hảufig und eingehend, aber stets mit durchaus negativem Resultat vorgenommenen Tintersuchungen gegeniiber verficht sie ihre Meinung in logischer, aber völlig einsichtsloser Weise, schildert Art und Sitz des Leidens mit größter Bestimmtheit, bringt alle sonstigen vorübergehenden Störungen ihres körperlichen Befindens, immer unter ausgesprochen hypochondrischer Deutung und laienhafter Konstruktion des $\mathrm{Zu}^{-}$ sammenhanges, zu dem vermeintlichen Grundleiden in Beziehung, hat für nichts als für dieses Interesse und hält sich für unrettbar verloren. Dabei besteht eine manische Lebhaftigkeit in ihrer Verteidigung der Beschwerden und ihren Dentungen und eine mehr leidenschaftlich-fanatische als depressive Stimmung. Nach einigen Monaten Wiederkehr der allgemeinen Interessen, milderes, zugänglicheres Wesen, allmähliches Zurücktreten der krankhaften Vorstellungen und Sensationen.

Meines Erachtens berechtigt nichts dazu, Fälle dieser Art als Neurasthenie zu bezeichnen. Ein zufälliges Ereignis - das Trauma - hat hier die habituell einseitig auf den eigenen Körperzustand gerichtete Aufmerksamkeit auf ein bestimmtes Organ - in diesem Fall den originär funktionsschwachell Darm - determiniert. Wir haben es mithin mit ideagenen Sensationen und Schmerzen zu tun, da hier „der hypochondrische Vorstellungsinhalt gewissermaben in rückläufiger Richtung den Gefühlston der Empfindung beeinflubt" und so "illusionäre Schmerzen und sogar halluzinatorische schmerzhafte Organempfindungen" (Binswanger) hervorgerufen hat. Es wäre aber auch nicht gerechtfertigt, Fälle dieser Art für das Bestehen einer selbständigen Krankheit Hypochondrie zu verwerten, da sie sich zwanglos der Gruppe der konstitutionellen Psychopathien einfügen lassen und insbesondere nahe Beziehungen zu einer unter diesen hervorragenden Krankheitsform, dem manisch-depressiven Irresein, besitzen.

Wir sind hiermit zu der Gruppe von Fällen gekommen, die in einer gewissen Phase ihres Verlaufs nicht selten den Anlaß zur irrtümlichen Diagnose einer Neurasthenie geben, während es sich tatsächlich um die depressive Phase des soeben erwähnten Krankheitsbildes handelt. Es ist vielleicht nicht unnötig, an dieser Stelle auf die heute wohl von del' Mehrheit der Fachgenossen acceptierte Lehre Kraepelins hinzuweisen, nach der wir in den scheinbar gegensätzlichen Zuständen manischer Gehobenheit und depressiver Hemmung Erscheillungsformen einer gemeinsamen Störıng zu sehen haben, der das klinische Bild des manisch-depressiven Irreseins entspricht. Dabei kann von einer wirklich streng periodischen Wiederkehr der einzelnen Anfälle nur in einer verschwindend kleinen Zahl von Fällen die Rede sein, meist ist bei den betreffenden Störungen vielmehr nur eille allgemeine Neigung, sich zu wiederholen, vorhanden, die der, allen Geistesstörungen aus krankhafter Veranlagung (Entartungsirresein, Epilepsie, Hysterie etc.) innewohnenden Eigenschaft, sich von Zeit zu Zeit in heftigeren Krankheitserscheinungen zu entladen, durchaus entspricht. Demnach kann es aber auch während des ganzen Lebens bei einem einzigen Anfall sein Bewenden haben, ohne daß dadurch die Zugehörigkeit des betreffenden Falles zu dieser Gruppe in Frage gestellt wüde. Was endlich die klinische Erscheinungsweise der einzelnen Anfälle dieses Leidens betrifft, so haben wir es wiederum Kraepelin zu danken, dab er uns von dem früher in dieser Beziehung herrschenden Schematismus losgemacht hat, der nur manische und melancholische Zustandsbilder miteinander abwechseln ließ, während tatsächlich in der klinischen Erscheinungsweise die größte Mannigfaltigkeit herrscht und nur gewisse regelmäßig vorhandene Grundstörungen das gemeinsame Band darstellen. Auch die lediglich durch depressive Bilder charakterisierten "zirkulären Depressionszustände" sind mit großer Wahrscheinlichkeit dieser Krankheitsform zuzurechnen.

Von den hierher gehörigen Depressionszuständen geben nun die leichten, meist außerhalb der Anstalt und vielfach auch außerhalb der psychiatrischen Behandlung ablaufenden 
Fälle häufig Anlaff zur Verwechslung mit Neurasthenie. Dies ist wiederholt, besonders von Hecker und von Kraepelin, betont worden, in weiteren Kreisen aber doch noch nicht hinreichend bekannt. Die betreffenden Kranken sind traurig verstimmt, arbeitsunlustig, entschlufunfähig, empfinden selbst die Erschwerung ihres Denkens, klagen über allerhand abnorme Sensationen, insbesondere Ermüdungsempfindungen, äufiern hypochondrische Befürchtungen, vielfach besteht auch Lebens überdruf. Von der Anführung von Beispielen hierfür kann abgesehen werden, dagegen sei ein Fall mitgeteilt, in dem zunächst lange Zeit nur solche leichte Depressionszustände auftraten, schlieblich aber eine ausgesprochene Psychose sich entwickelte, die wohl als atypischer Fall von manisch-depressivem Irresein aufzufassen ist.

Der Kranke, ein jetzt etwa fünfzigjähriger, feingebildeter Mann, erblich belastet und selbst von jeher ängstlich, an verschiedenen "Phobien" (z. B. beim Aufenthalt in größeren Menschenmassen, an hachgelegenen Plätzen, beim Eisenbahnfahren, beim Schwimmen in tiefem Wasser) leidend, wird Anfang der dreiBiger Jahre nach Ueberarbeitung und Sorge (frühere Infektion) schlaflos, reizbar, neurasthen is ch; Ermüdungsgefuhle, Blendungserscheinungen, Kopfdruck, Schwindel-, Angstempfindungen und Herzbeschwerden treten bei geringfügigen Anlässen auf. Depressive Verstimmungen mit Selbstanschuldigungen und hypochondrischer Selbstbeobachtung. In den folgenden Jahren sehr ungleiches Verhalten, meist im Winter günstigeres Befinden, dann sogar lebhafter Trieb zur Tätigkeit, zu anderen Zeiten unfăhig zur Arbeit, immer leicht ermüdbar und unfähig, sich längere Zeit zu konzentrieren. Fast alljährlich Aufenthalt in Sanatorien, die er meist gebessert verläBt. Im Jahre 1902/03 Verschlechterung des Zustandes; Ohnmachts-, Erschöpfungsgefühle, Unfähigkeit zu gehen (psychogen) rufen die Befürchtung, sterben zu müssen, hervor quälende, obscöne Zwangsgedanken. Beim Eintritt in die psychiatrische Klinik (Juli 1903) s chwerer Depressionszustand. Zahlreiche unangenehme Sensationen (Blitzen vor den Augen, Glühen des Körpers, Rückenschmerz, Gefühl des Schwankens, Schwebens), hypochondrische Wahnideen (Gehirn und Ruckenmark seien mit dem Urin abgeflossen, der ganze Körper sei verändert, er selbst ganz blödsinnig) und melancholische Selbstanklagen (er sei eitel, egoistisch, schuld an allem Elend, habe die Welt vergiftet, Kaiser und Volk zugrunde gerichtet). Dazu zwangs mäBige Gotteslästerungen. Zeitweise hypochondrische Gehstörung. He mmung von wechselnder Stärke, spricht meist zögernd, leise; kann sich zu keinem EntschluB aufraffen. Halluzinationen spärlich zugegeben (Gottes Stimme, metallischer Geschmack im Munde). - Allmählich etwas lebhafter und freier, aber immer deutlich gehemmt. Dann wieder tiefere Depression. In sich versunken, Zwangs- und Wahnvorstellungen wie oben. Dann allmählich mitteilsamer, berichtet von Beeinträ chtigungen (,man" macht ibm die Gedanken, gibt ihm bestimmte schädliche Stoffe, will ihn verderben), sagt aber nicht, von wem diese ausgehen.

Etwa April 1904 Umschlag der Stimmung und aus gesprochen manisches Bild. das nun in etwas wechselnder Intensität mehrere Monate anhält: Der Kranke ist ganz im Gegensatz zu seinem bisherigen Verhalten sehr aktiv, beantwortet ohne Aufforderung seine monatelang liegen gelassenen Briefe, schreibt lange Berichte über sein Befinden, holt geschüftliche Auskünfte ein, denkt sich technische Verbesserungen aus, die er alsbald patentieren lassen will, macht Bestellungen und Einkäufe, gibt unnötig Geld aus, zeigt gesteigertes Selbstgefühl und rühmt sein Wohlbefinden, seine Kraft, seine neu entdeckten Fähigkeiten. Auch auf sein Aeußeres legt er viel größeren Wert, macht Heiratspläne etc. Bei seinen Beschäftigungen sehr unbestandig, fängt bald dies, bald jenes an, um es bald wieder aufzugeben. In seinen schriftlichen Auslassungen sehr weitschweifig, flüchtig. Dabei hält er an den früher nur angedeuteten Beeinträchtigungsideen durchaus fest, während die anderen depressiven Vorstellungen vollkommen verschwunden sind. Ueber erstere äußert er sich ebenso wie über seine massenhaften Sinnestäuschungen in bereitwilligster und ausführlichster Weise schriftlich und mündlich: Die Pfleger bestehlen ihn, geben ihm Gift im Essen, verderben seine Zeichnungen, tragen seine Kleider; spürt überall elektrische Beeinflussungen, spricht von gegen ihn als Medium geleiteten Strömen, von politischen Umtrieben. Er hört beleidigende Stimmen, sieht Gesichter, Bilder etc., phosphoreszierende Gebilde, klagt über Blendungserscheinungen, Doppelsehen etc. er riecht Chloroform, Formalin, entdeckt Bromsilber in der Luft; endlich leidet er unter zahlreichen unangenehmen Sensationen an den Genitalorganen, dem After und an anderen Körperstellen, die er auf elektrische Beeinflussungen zuräckführt. Im Bemühen, sich gegen diese zu schützen, entwickelt er eine sehr große Finergie und Vielgeschäftigkeit, stopft sich die Ohren zu, hängt Tafeln mit be- ruhigenden Farben auf, verhängt das Fenster mit gränen Vorhängen, leitet durch Kupferdrähte ab und trifft zahlreiche andere, zum Teil seltsame Veranstaltungen Alle diese Machinationen führt er auf das Ptlegepersonal zurück, dem er schroff begegnet; queruliert beständig, macht lange Anzeigen gegen die Pfleger, zeigt sich aber den Aerzten gegenüber stets liebenswürdig, dankbar, vertrau. ensvoll, benimmt sich überhaupt ganz korrekt, ist durchaus natürlich, sehr angeregt und interessiert. Nach mehrmonatigem Bestehen der genannten Störungen wird er allmählich ruhiger; entfernt alle Schutzmaßregeln aus seinem Zimmer, schreibt wenig, queruliert and beschwert sich nicht mehr, wird höflich gegen das Pflegepersonal. Die abnormen Sensationen und die Halluzinationen bestehen dabei noch fort, werden aber allmahlich von ihm selbst immer deutlicher als krankhaft erkannt. So entwickelt sich ein $\mathrm{Zu}$ stand, in dem Patient den Eindruck eines völlig gesunden, sehr intelligenten Mannes macht, abgesehen von einer dauernd bestehen. den, leichten Ermüdbarkeit und Neigung zu vermehrter Selbstbeobachtung. Auf Befragen gibt er allerdings immer noch das $\mathrm{Be}-$ stehen unangenehmer Sensationen und $Z$ wangsvorstellungen an, die in ihrer Intensität wechseln; auch von Stimmen, die er meist als Gedankenlautwerden und als seine Handlungen begleitende kritische Bemerkungen schildert, will er niemals frei gewesen sein und auch jetzt noch solche hören. Er erklärt diese aber selbst aus seinem krankhaften Zustande und knüpft daran weder wahnhafte Deutungen, noch läpt er sich dadurch in seinem Benehmen irgendwie beeinflussen. - In neuester Zeit etwas stärkere Schwankungen der Arbeitsfahigkeit und Stimmung, die er auf vermehrte Sensationen zurückführt. Patient befindet sich noch in Behandlung.

Im Vergleich mit so komplizierten Bildern wie dem soeben mitgeteilten, erscheinen die geistigen Störungen bei der "einfachen" Neurasthenie, wie wir sie oben abgegrenzt haben, verhältnismäßig einfach. Sie betreffen die Stimmung, die Vorstellungstätigkeit und auch das Handeln der Kranken. Es ist ohne weiteres verständlich, daß die unverhältnismäfig lebhaften und peinlichen Empfindungen, mit denen sie infolge der bestehenden psychischen Hyperalgesie auf geringfügige, selbst physiologische Reize reagieren, die Gemütslage nachhaltig beeinflussen müssen. Diese Stimmungsanomalie wird durch die Bezeichnung „reizbare Verstimmung " ganz gut charakterisiert; die Kranken sind mifmutig, unbefriedigt, verdrieflich, apathisch, grüblerisch, dabei aber aus geringfügigstem Anla $B$ aufbrausend und $\mathrm{zu}$ pathologischen Affekterregungen geneigt. Insbesondere sind es die den Denkprozeb selbst begleitenden Ermüdungs- und Schmerzempfindungen und das daraus entspringende Gefühl der geistigen Leistungsunfähigkeit, welche eine mutlose, verzagte Stimmung unterhalten. Ebenso sind die krankhaften Empfindungen die Quelle der häufigen nosophobischen oder hypochondrischen Vorstellungen, die zwar meist nur den Charakter unbestimmter Befürchtungen haben, episodisch aber auch nicht selten zu festen Ueberzeugungen werden. Im übrigen sind aus dem Gebiet der Vorstellungstätigkeit zu erwähnen, in inhaltlicher Beziehung die auch bei der einfachen Neurasthenie zuweilen auftretenden Zwangsvorstellungen, in formaler die auf eine Erleichterung oder Erschwerung des Gedankenablaufs hindeutenden Symptome (Gedankenjagd, Zwangsdenken einerseits, Unfähigkeit zu anhaltender geistiger Beschäftigung, Vergeßlichkeit bis zu völligem Versagen der psychischen Leistungen anderseits). Von ganz besonderer praktischer Wichtigkeit sind endlich die Angstgefühle und Angstanfälle der Neurastheniker, die entweder ohne ursächliche Verbindung mit dem Vorstellungsinhalt, etwa durch eine krankhafte Empfindung, eine Gemütsalteration, eine einfache Gedankenoperation etc., ausgelöst und nachträglich im Sinne hypochondrischer Befürchtungen motiviert werden oder sich erst an Vorstellungen, die gleichfalls meist nosophobischen Inhalt haben, anschlieBen. Die Angst wird zuweilen als eine allgemeine geschildert, bemerkenswert sind aber die verschiedenen Lokalisationen, welche die Kranken ihr subjektiv zu geben pflegen, indem sie sie in die von ihnen als Sitz ihrer krankhaften Empfindungen aufgefaßten Körperregionen verlegen. (Herzgegend, Kopf, Brust, Bauch, Extremitäten.) Diese Angstanfälle, die bei Neurasthenikern, dem vielfachen unmotivierten Wechsel der Krankheitserscheinungen entsprechend, plötzlich auftreten und die Besonnenheit der Kranken überwältigen können, führen nicht selten zu triebartigen Selbstmordversuchen.

Die soeben gegebene Schilderung ist durchaus skizzenhaft 
und lediglich dazu bestimmt, zu zeigen, dafl auch die ausgesprocheneren geistigen Anomalien bei der Neurasthenie ihre Wurzel in den $\mathrm{krankhaften} \mathrm{Empfindungen} \mathrm{haben.} \mathrm{Zwischen}$ den Fällen, in denen uns das psychische Moment bei der Neurasthenie lediglich in Form der elementaren Empfindungsstörung entgegentritt, und jenen, in denen man mit Recht von einer neurasthenischen Geistesstörung sprechen kann, liegen aber Abstufungen aller Grade und sind also nur quantitative Unterschiede vorhanden Es wäre hiernach ein müBiges Beginnen, zu untersuchen, wo bei der Neurasthenie die Neurose aufhört und die Psychose anfängt, da es eine scharfe Grenze hier nicht geben kann. 